\title{
Scrambling the egg origin dogma
}

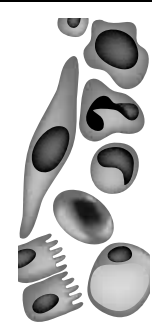

\author{
PTalbot ${ }^{\text {lt }} \&$ \\ L Femander ${ }^{1}$ \\ ${ }^{\dagger}$ Author for correspondence \\ ${ }^{1} \mathrm{D}$ epartment of Cell Biology \\ and N euroscience, U niversity \\ of California, Riverside, \\ CA 92521, U SA \\ Tel.: +1 9518273768 ; \\ Fax: +1 9518274286 \\ E-mail: talbot@ucr.edu
}

\begin{abstract}
Evaluation of: Eggan K, Jurga S, Gosden R, Min IM, Wagers AJ. Ovulated oocytes in adult mice derive from non-circulating germ cells. Nature 441, 1109-1114

(2006) [1]. This interesting study investigates the hypothesis that stem cells in adult bone marrow and the circulatory system populate mouse ovaries and contribute to the germline. When pairs of GFP ${ }^{+}$and GFP- parabiotic mice with joined circulatory systems were superovulated after 8 months of parabiosis, oocytes collected from the oviducts had the phenotype of the animal from which they had come. Follow-up experiments, in which oocytes were chemically destroyed before establishing parabiosis, yielded similar results. Injection of GFP+or GFP- bone marrow cells into mice with ovaries ablated using ovotoxins or irradiation did not rescue production of ovulated oocytes. These data do not support the controversial hypothesis that bone marrow stem cells contribute to the germline of adult female mice.
\end{abstract}

For over 50 years, the idea that mammalian ovaries contain a finite number of oocytes at birth has been a dogma [2]. This concept was seriously challenged in 2004, when Jonathan Tilly's laboratory at $\mathrm{H}$ arvard University (M A, USA) demonstrated that mouse ovaries may renew their supply of oocytes during adulthood [3]. U sing a combination of experimental approaches, Tilly's group concluded that mouse ovaries would run out of oocytes owing to atresia shortly after entering puberty if the population were not replenished. In a follow-up study, Tilly's group went on to show that, if oocytes were chemically destroyed, intravenous injection of bone marrow cells from adult females resulted in the appearance of several hundred small follicles that presumably came from stem cells in the bone marrow transplant. M ice with a targeted disruption in the ataxia telangiectasia gene, which are sterile and have few (if any) oocytes, also acquired follicles following bone marrow transplants or injection of peripheral blood cells. In the peripheral blood rescue experiment, some follicles with green fluorescent protein (GFP)+ oocytes, indicative of blood cell incorporation into the ovary, were observed within $24 \mathrm{~h}$ of the transplants. These findings were so unorthodox that numerous reviews immediately challenged the concepts conveyed in the Tilly papers (e.g., [4,5]).

Follow-up studies in other laboratories on this interesting discovery have been slow in coming. However, this June, a publication addressing oocyterenewal in mice was published in $\mathrm{N}$ ature[1].
This ingenious study from Amy Wagers' laboratory (also at $\mathrm{H}$ arvard U niversity) used the interesting method of parabiosis, in which a pair of mice share their circulatory systems, to determine if circulating stem cells could pass between mice, colonize ovaries and develop into ovulated oocytes. O ne mouse in each parabiotic pair was transgenic for GFP, which it expressed in every cell, while the other mouse was wild-type, without any GFP expression. If stem cells from the bone marrow could enter the peripheral circulation and be transferred between animals and if these stem cells could be incorporated into the ovary and develop into oocytes, one would expect to see GFP+ oocytes in wild-type mice and GFP- oocytes in transgenic females. To assay for stem cell transfer to ovaries, Wagers' laboratory induced superovulation in females, collected oocytes from the oviduct and assessed them for chimerism using fluorescent microscopy.

\section{Results}

When parabiotic mice were joined together at 4-8 weeks of age and remained coupled for 6-8 months, extensive chimerism was found in the peripheral blood and hematopoietic stem cell populations of both transgenic and wildtype mice, demonstrating that their circulatory systems were indeed coupled. While G FP+ cells of the hematopoietic lineage were occasionally found in cumulus masses of wild-type females, only $\mathrm{GFP}^{+}$oocytes were recovered from transgenic animals and only GFP- oocytes were 
recovered from their nontransgenic partners. Since Tilly's experiments involved chemical destruction of oocytes, Wagers' group reasoned that damage to the mouse tissues could affect the experimental outcome. They next treated mice with ovotoxins (cyclophosphamide [Cy] and busulfan [Bu]) to destroy oocytes and then created parabiotic pairs. After both 2 weeks and 2 months of parabiosis, oocytes collected from superovulated females always had the GFP phenotype of the animal from which they came. A total of 665 oocytes were examined from parabiotic pairs in this study, and none had chimeric phenotypes.

In a final experiment, mice were treated with ovotoxins or $\gamma$-irradiation to destroy oocytes, followed by intravenous injection with GFP+ or GFP- bone marrow from donor mice. This experiment tested the possibility that bone marrow stem cells may not have entered the circulation and reached the ovaries in the parabiotic pairs studied previously. Animals receiving $\gamma$-irradiation ovulated no oocytes, indicating that injected marrow cells had not rescued production of oocytes capable of ovulation. Animals treated with ovotoxins ovulated reduced numbers of oocytes that were invariably of the phenotype of the animal from which they came, in agreement with the parabiotic studies. The failure to rescue oocyte production and the absence of oocyte chimerism in these experiments support the conclusion that the bone marrow does not contribute to oocyte renewal in adult mice.

\section{Significance \& perspective}

The results of the Wagers' paper appear to refute Tilly's studies. H owever, there are several differences in the experiments that make direct comparisons difficult. For example, the assays used in the two laboratories were not the same. Wagers and colleagues assayed ovulation of oocytes, while Tilly's group assayed appearance of immature follicles in ovaries. O vulation is a valuable assay demonstrating that a fully mature, presumably functional, oocyte has been produced; although assaying ovulation would not necessarily catch the production of the immature follicles reported by Tilly's group. It is possible that bone marrow stem cells populate the ovary, differentiate into immature oocytes, but never fully mature and ovulate or that their ovulation is such a rare event that it was not caught in the Wagers study, even though a seemingly large number of oocytes was examined. It would be interesting to know if the parabiotic mice had immature oocytes that were chimeric but became atretic before having the opportunity to ovulate.

Several other differences may also be important when comparing these studies. The mouse substrains used by the two groups were different (Tilly used C57BL/6, while Wagers used $\mathrm{C} 57 \mathrm{BL} / \mathrm{Ka}$ ) and genetic differences may have played a role in the experimental outcome. In addition, Tilly's group injected antibodies to CD 4 and CD 8 prior to bone marrow transplants, while Wagers group apparently did not. These antibodies shut down T-lymphocyte responses, which are critical for long-term immunity and rejection of transplants. Since the ability of multipotent adult progenitor cells to survive after a transplant is diminished by the persistence and activity of $T$ and B cells [6], Tilly's use of additional immunosuppressants may have favored survival and differentiation of bone marrow stem cells in the ovary. The bone marrow transplants themselves were not equivalent. While Wagers' lab used whole bone marrow, Tilly's laboratory used a fraction of pluripotent bone marrow cells that was enriched for germline markers and lacked hematolymphoid commitment, which may have favored engraftment of transplanted cells in the ovary. Finally, the routes of bone marrow injection, which can affect engraftment of marrow cells [6], also differed in that Wagers used retro-orbital injection and Tilly used intravenous tail injection. This difference in injection sites could also explain why Wagers observed low-level hematolymphoid chimerism in the bone marrow recipients.

Two highly toxic chemicals, $\mathrm{Cy}$ and Bu were used by both laboratories, in the same dosages, to chemoablate ovaries. In Tilly's peripheral blood transplant experiments, engraftment of cells was evaluated using a GFP marker. Curiously, a nonablated control group was not included, even though $\mathrm{GFP}^{+}$-engrafted cells would have been detectable in ovaries containing endogenous follicles. Without this control, it is not clear if the results that Tilly obtained require chemoablation. Would peripheral blood cells engraft in ovaries that were not ablated by $\mathrm{Cy}$ and Bu? This seems an important question that needs to be answered.

In a related study using the same mouse strain as Tilly, the number of healthy ovarian follicles was counted in mice between days 1 and 200 after birth [7]. Although there was an overall decrease in follicle number during this interval, the number of follicles did not decline 
between days 7 and 100. These authors did not look directly for a stem cell population in the ovaries, although their data do support the idea that follicle renewal occurs in mice, and appear to support the premise in Tilly's 2004 paper [2].

The experiments in the Tilly and Wagers papers are fascinating, well presented and make very good reading. It is too soon to understand why their results do not agree. N evertheless, they have certainly opened up an exciting area for future research and illustrate very clearly that we have a lot yet to learn regarding the mammalian ovary and production of oocytes. W hile bone marrow plasticity is a controversial topic [8], a recent study from $\mathrm{G}$ ermany provides evidence that bone marrow stem cells can transdifferentiate into male germ cells [9], an observation that seems to keep alive the possibility that oocytes could also differentiate from marrow stem cells.

\section{Executive summary}

- Parabiosis (anastomosis of circulatory systems in two adult mice) was used to examine the hypothesis that stem cells from the bone marrow can enter the peripheral circulation, populate adult ovaries and contribute to the germline.

- Transfer of cells between mice was monitored by having one transgenic mouse (GFP+ in every cell) in each parabiotic pair and one wild-type (GFP-) mouse.

- Chimerism was found in the blood cells after 8 months of parabiosis (wild-type mice had GFP+ blood cells and transgenic mice had GFP- blood cells); however, the authors found no chimerism in ovulated oocytes.

- In follow-up experiments, chemicals were used to deplete ovaries of oocytes, and oocytes from parabiotic pairs again had the phenotype of the animal from which they had come.

- Finally, injection of bone marrow cells from GFP+ or GFP- mice could not rescue oocyte production (as assessed by ovulation) following ablation of oocytes with ovotoxins or $\gamma$-irradiation.

- These data do not support the hypothesis that bone marrow contributes to the ovarian germline in adult mice. Since the authors monitored ovulation and did not look for immature oocytes, the data in this study cannot be compared directly with prior work from Tilly's laboratory that does support the hypothesis.

\section{Bibliography}

1. Eggan K, Jurga S, Gosden R, M in IM, Wagers AJ: $O$ vulated oocytes in adult mice derive from non-circulating germ cells. Nature 441, 1109-1114 (2006).

2. Zuckerman S: Thenumber of oocytes in the mature ovary. Recent Prog. H orm. Res. 6 , 63-108 (1951).

3. Johnson J, Canning J, Kaneko T, Pru JK, Tilly JL: Germline stem cells and follicular renewal in the postnatal mammalian ovary. N ature 428, 145-150 (2004).
4. Byskov AG, Faddy M J, Lemmen JG, Andersen $C Y$ : Eggs forever? D ifferentiation 73, 438-446 (2005).

5. Telfer EE, Gosden RG, Byskov AG et al.: $O n$ regenerating the ovary and generating controversy. Cell 122, 821-822 (2005).

6. Tolar J, 0 'Shaughnessy JM , Panoskaltsis-M ortari A et al.: H ost factors that impact the biodistribution and persistence of multipotent adult progenitor cells. Blood 107, 4182-4188 (2006).

7. Kerr JB, D uckett $R, M$ yers $M, B$ ritt $K L$, M ladenovska T, Findlay JK: Q uantification of healthy follicles in the neonatal and adult mouse ovary: evidence for maintenance of primordial follicle supply. Reproduction 132, 95-109 (2006).

8. Wagers AJ, Sherwood RI, Christensen JL, Weissman IL: Little evidence for developmental plasticity of adult hematopoietic stem cells. Science 297, 2256-2259 (2002).

9. N ayernia $\mathrm{K}, \mathrm{N}$ olte J, M ichelmann $\mathrm{H}$ W et al.: In vitro-differentiated embryonic stem cells give rise to male gametes that can generate offspring mice. D ev. Cell 11, 125-132 (2006). 